\title{
A Fairness-Based Access Control Scheme to Optimize IPTV Fast Channel Changing
}

\author{
Junyu Lai, ${ }^{1}$ James C. F. Li, ${ }^{1}$ Alireza Abdollahpouri, ${ }^{2}$ Jianhua Zhang, ${ }^{3}$ and Ming Lei ${ }^{1}$ \\ ${ }^{1}$ NEC Labs China, Beijing 10084, China \\ ${ }^{2}$ Department of Computer Engineering and Information Technology, University of Kurdistan, Sanandaj 66177 15175, Iran \\ ${ }^{3}$ College of Computer Science, Zhejiang University of Technology, Hangzhou 310023, China \\ Correspondence should be addressed to Junyu Lai; laijyl@gmail.com
}

Received 2 April 2013; Revised 11 November 2013; Accepted 26 November 2013; Published 4 February 2014

Academic Editor: Dongdong Ge

Copyright (c) 2014 Junyu Lai et al. This is an open access article distributed under the Creative Commons Attribution License, which permits unrestricted use, distribution, and reproduction in any medium, provided the original work is properly cited.

\begin{abstract}
IPTV services are typically featured with a longer channel changing delay compared to the conventional TV systems. The major contributor to this lies in the time spent on intraframe (I-frame) acquisition during channel changing. Currently, most widely adopted fast channel changing (FCC) methods rely on promptly transmitting to the client (conducting the channel changing) a retained I-frame of the targeted channel as a separate unicasting stream. However, this I-frame acceleration mechanism has an inherent scalability problem due to the explosions of channel changing requests during commercial breaks. In this paper, we propose a fairness-based admission control (FAC) scheme for the original I-frame acceleration mechanism to enhance its scalability by decreasing the bandwidth demands. Based on the channel changing history of every client, the FAC scheme can intelligently decide whether or not to conduct the I-frame acceleration for each channel change request. Comprehensive simulation experiments demonstrate the potential of our proposed FAC scheme to effectively optimize the scalability of the I-frame acceleration mechanism, particularly in commercial breaks. Meanwhile, the FAC scheme only slightly increases the average channel changing delay by temporarily disabling FCC (i.e., I-frame acceleration) for the clients who are addicted to frequent channel zapping.
\end{abstract}

\section{Introduction}

A vitally important factor leading IPTV services to be successful is to ensure clients' quality of experience (QoE) to be better than or at least comparable to the conventional TV systems. However, IPTV systems are generally featured with a longer channel changing delay, which is around 2 seconds, and can lengthen from 5 to 10 seconds with next generation video coding paradigms. This unfavourable feature can fatally degrade clients' QoE and, consequently, incur revenue loss for IPTV service providers. TV channel changing delay is defined as the latency between the initiation of changing to a targeted channel (e.g., by pressing the buttons on the remote control) and the final display of the content on that channel. In a typical IPTV system, channel changing delay consists of several different components, which are summarized in Table 1. A representative mean value for each component is also given based on previous literature and practical measurements [1]. It is obvious that the latency owing to Intraframe (I-frame) acquisition is the dominant factor contributing to the total channel changing delay. Iframe acquisition delay is directly related to the compression schemes used in modern video codec technologies (such as MPEG-2 or MPEG-4). More specifically, pictures in a video stream are encoded into either intracoded mode (i.e., I-frame) or interprediction mode (i.e., P- or B-frame). I-frames do not rely on any other frame for decoding, while the decoding of a $\mathrm{P}$ - or B-frame depends on the neighbouring frame(s). Owing to the fact that the size of an I-frame is typically much larger than that of a P- or B-frame, the frequency of the I-frames in a video stream should be small enough for an efficient compression scheme. Also note that when a user switches into a specific channel the upcoming I-frame should be the first independently decodable frame. Thus, we denote the time a user has to wait until the next I-frame arrives as I-frame acquisition delay.

In order to decrease the IPTV channel changing delay, various fast channel changing (FCC) methods are proposed. 
TABLE 1: Major components of channel changing delay.

\begin{tabular}{lc}
\hline Delay component & Typical latency $(\mathrm{ms})$ \\
\hline $\begin{array}{l}\text { IGMP session joining/leaving for } \\
\text { multicast delivery }\end{array}$ & $\sim 100$ \\
Network latency and jitter & $\sim 100$ \\
Client stream buffering (network dejitter & $\sim 250$ \\
buffer and video input buffer) & $\sim 1000$ (GOP duration \\
I-frame acquisition & is 2 seconds) \\
Video/audio synchronization & $\sim 200$ \\
Content authorization & $\sim 100$ \\
\hline
\end{tabular}

Among them the most widely adopted ones in practical engineering are based on the so-called I-frame acceleration mechanism. The IPTV system promptly transmits to a user conducting channel changing a retained I-frame of the requested channel as an (accelerated) uncasting communication, so as to reduce user waiting time for the first picture of the targeted channel. However, this mechanism has an inherent scalability problem which can be aggravated by the explosions of channel changing requests during commercial breaks. In particular, the scalability problem refers to the case that the network bandwidth required by using this I-frame acceleration mechanism can be considerably larger than the available bandwidth, which accordingly introduces heavy degradations to network quality of service (QoS) and user QoE. In order to mitigate the problem, we propose a fairness-based admission control (FAC) scheme as a scalability optimization method for the I-frame acceleration mechanism. Based on the channel changing histories of all the clients, our proposed FAC scheme can intelligently decide whether or not to conduct Iframe acceleration at each arrival of channel change requests. We carry out comprehensive simulation experiments, which demonstrates the potential of our proposed FAC scheme to optimize the scalability of the most widely used I-frame acceleration mechanism. Besides, the disadvantage brought by using the FAC scheme is also investigated.

The rest of the paper is organized as follows. Section 2 presents the state-of-the-art. More detailed introduction of the I-frame acceleration mechanism as well as its shortcomings is introduced in Section 3. Our major contribution is illustrated in Section 4, where we propose a FAC scheme in order to optimize the scalability of the aforementioned Iframe acceleration mechanism. Comprehensive simulation experiments have been conducted to evaluate the performance of the FAC scheme in Section 5. Finally, summary and outlook are given in Section 6.

\section{Related Work}

As aforementioned, a short channel changing delay is one of the key factors for IPTV services. In both academia and industry, there exist a plenty of research efforts to reduce the delay associated with different parts of channel changing process (such as those listed in Table 1). In [2], Cho et al. proposed an approach based on the prediction that the adjacent channels are more likely to be watched by viewers (choosing channel by UP and DOWN buttons on the remote control) for the next time of channel changing. Their method requires to conduct the simultaneous transmission of the two adjacent channels. Soon after that, an improved approach was introduced in [3], where a statistical selection of channels are derived based on the historical channel changing information of each user, which is more accurate than always choosing the adjacent channels. However, the disadvantage lies in the fact that the bandwidth demand is proportional to the number of channels being concurrently transmitted and, consequently, the bandwidth demands will be tripled if these methods are applied. In order to mitigate the delay introduced by IP multicasting, [4] developed the sourcespecific multicast (SSM) mechanism to significantly reduce the complexity of IP multicasting routing process. There are a set of schemes $[5,6]$ aiming to decrease the I-frame acquisition latency based on adding a channel change tune-in stream, in addition to the main stream, for each provided TV channel. More specifically, the tune-in stream can be either a low-resolution (I-frame only) stream [5] or a full resolution (I-frame only) stream [6]. However, the former scheme can only provide a lower resolution before a full resolution Iframe arrives on the main stream, and the latter scheme may introduce prediction mismatches for the $\mathrm{P}$ - or B-frames in the main stream if the prediction is based on an I-frame in the tune-in stream. Similar to the low-resolution tune-in stream scheme, scalable video coding (SVC) [7] technology can also be employed to reduce the channel changing delay by using the base layer as the tune-in stream. The only difference lies in the fact that, after turning to the enhancement layer stream, the base layer stream is still used together with the enhancement layer stream to produce a full resolution video. In practice, the most widely used methods (e.g., Microsoft's FCC [8]) are based on the I-frame acceleration mechanism. As already mentioned, this mechanism promptly transmits to a user conducting channel changing a retained I-frame of the requested channel, aiming to reduce the latency for displaying the first picture of the targeted channel. This method is based on IP unicasting, which may consume a large amount of network bandwidth. In order to mitigate this problem, IP multicasting based I-frame acceleration mechanism was proposed in [9]. As a summary, each of the above approaches has its advantages and application limits. In general, there exist trade-offs between the considered channel changing delay and some other important performance metrics, for example, the consumed bandwidth.

As opposed to conventional TV systems, IPTV user behaviour can directly influence the traffic loads on the transmission networks. A large amount of existing research efforts have been conducted on the IPTV traffic characterization as well as user behaviour measurement, analysis, and modelling. In [10] Qiu et al. studied a large national IPTV system and developed a series of analytical models for different aspects of user activities. Ramos et al. [11] modelled the behaviour of a typical user as a rapid burst of channel selection events followed by an extended period of channel viewing. Yu et al. [12] investigated IPTV user activities in terms of zapping rate and session lengths. Cha et al. [13] studied how users select 


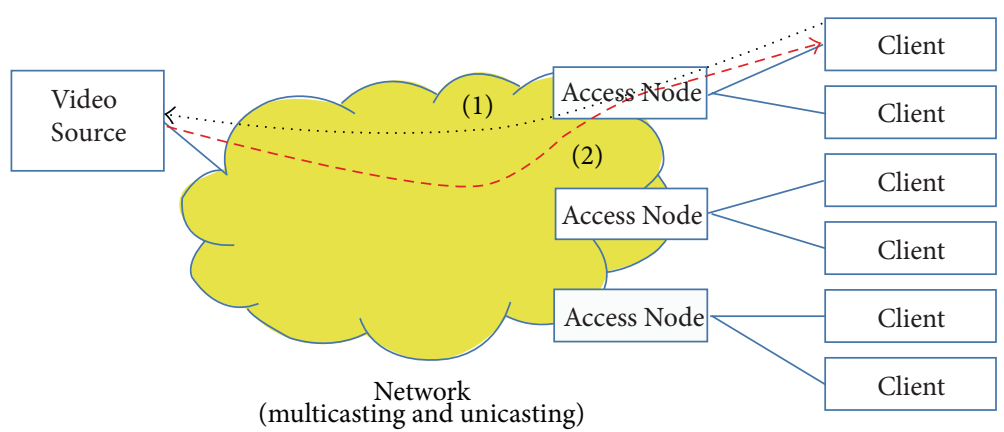

(1) Channel request from a client

(2) Multicast the requested channel

FIGURE 1: A typical IPTV distribution architecture.

channels in the real world based on a comprehensive trace from a commercial IPTV service, including channel popularity dynamics, aggregate viewing sessions, and content locality. In our previous work $[14,15]$ (which was an extension of earlier IPTV user behaviour models), we modelled the channel switching behaviour of a single IPTV user. Both channel popularity and user activities have been taken into account. Our proposed IPTV user behaviour automaton (IPTV-UBA) model relies on formal descriptions to capture the characteristics of user behaviours. The aggregate user behaviour can be derived by overlaying the behaviours of a set of users.

\section{The Most Widely Used Fast Channel Changing Mechanism and Its Shortcomings}

As summarized in the previous section, a plenty of research efforts have been carried out to improve the QoE for IPTV clients by shortening the channel changing delay from different perspectives regarding the factors listed in Table 1. Due to the fact that I-frame acquisition delay usually has the largest contribution to the total delay, the most effective and practically deployed fast channel changing methods are based on the I-frame acceleration mechanism. This mechanism was firstly proposed by Microsoft [8] and soon afterwards other companies (such as Huawei, ZTE, and Cisco) published their own improvements. In this section, we first present the principle behind the I-frame acceleration mechanism. Then, we discuss the shortcomings of using this mechanism and analyse the reasons.

3.1. I-frame Acceleration Mechanism. In a typical IPTV distribution architecture as illustrated in Figure 1, there exists a Video Source responsible for transmitting all the live TV channels to its downstream Clients by means of dynamic or static multicasting via the Network in between. The network in the architecture contains a plurality of replication nodes (such as aggregation routers and switches), which forms a hierarchical networking architecture (e.g., tree topology) and supports both multicasting and unicasting communications. The simplified process of a user carrying out a new channel change is marked in Figure 1. A client in the first step sends a channel change request to the Video Source. After the request is approved, the Video Source starts to multicast the requested channel to the client in the second step. In fact, the multicasting joining process corresponding to the channel changing can usually be accomplished by the closest intermediate node to the client in the transmission path, where the requested channel is already available. It is worth noting that in this scenario all the TV channels are transmitted by means of IP multicasting, and the channel changing delay due to I-frame acquisition can be considerably large and variable as discussed in Section 1.

In order to reduce the channel changing delay and improve the QoE for clients by applying the I-frame acceleration mechanism, a channel change server (CCS) is introduced in the architecture presented in Figure 1 (see Figure 2). Depending on various system implementation features such as the scale of an IPTV system, the location of a CCS can be either at the edge of the core network or within a point-ofpresence $(\mathrm{PoP})$. The CCS is configured to retain at least the most recent I-frame, that is, the one just transmitted before the current instant, for each IPTV channel, and is adapted to respond to channel change requests from clients by transmitting the retained I-frame of a requested TV channel using an IP unicasting communication (probably with a higher transmission bit-rate than the average multicasting bit-rate), so that clients can receive the first picture of the requested channel much faster. Figure 2 demonstrates the service procedure when a client conducts the channel changing with applying the aforementioned I-frame acceleration mechanism. As can be seen, in the first step, a channel change request from a client is directly sent to the CCS. Then, upon receiving the request the CCS immediately transmits the retained I-frame of the requested channel back to the client by means of IP unicasting. Next, the client initiates the multicasting joining process to the requested TV channel towards the Video Source. At last, if successfully joining into the multicasting group, the client starts to receive the IP multicasting flow of the requested channel.

3.2. The Shortcomings of the I-Frame Acceleration Mechanism. It can be observed from practical IPTV systems that channel changing events are usually correlated and aligned with the timeline of TV programs. More specifically, at the end of each TV program (or during the commercial breaks), a large 


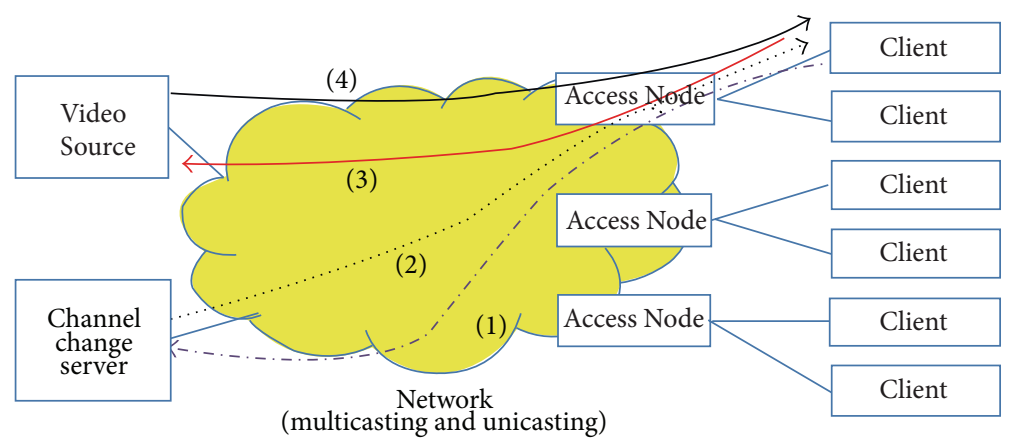

(1) Channel request from a client

(2) Unicast the retained I-frame for FFC

(3) Conduct the multicast joining

(4) Multicast the requested channel

FIGURE 2: A typical IPTV distribution architecture with CCS.

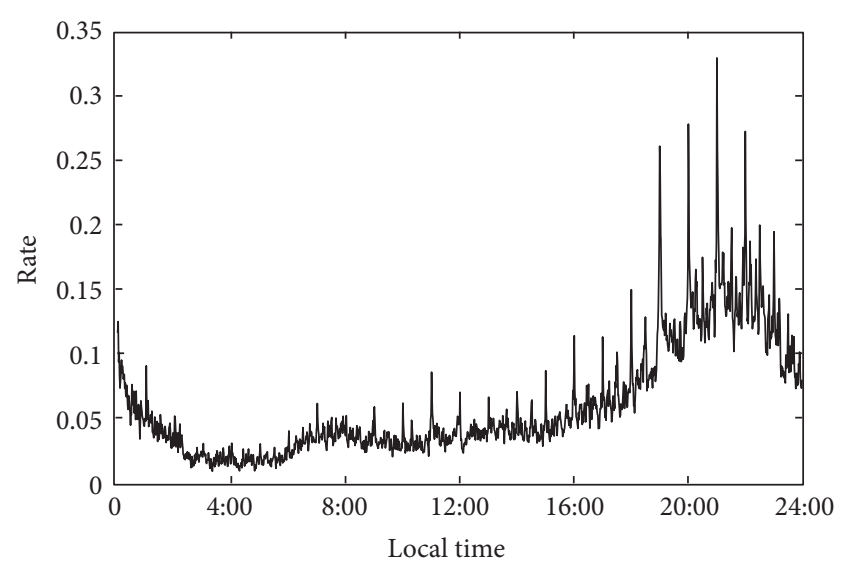

FIGURE 3: An illustration of periodic bursts of channel zapping events (derived from [10]).

portion of online clients tend to conduct continuous channel zapping for a short period of time (e.g., several minutes), with the purpose to search for the channels of interest. In addition, we have also noticed the fact that the ends of the TV programs (or the starts of the commercial breaks) on different channels are generally aligned at every half an hour. Therefore, from the perspective of the entire IPTV system, the rate of channel changing events is likely to periodically burst up to double or even triple of its average value, as is illustrated in Figure 3 (derived from [10]). Figure 3 presents the diurnal change of the aggregate channel changing events measured in a national-wide IPTV system with over two million clients in a representative working day. Particularly, it shows the timeline of these bursts, which are defined as the total number of channel changes normalized by the number of online clients. We can observe very strong spikes with the period of every 30 minutes, which reflect the correlated user activities due to the temporary alignment of TV programs.

As already indicated, when the I-frame acceleration mechanism is used, a separate IP unicasting stream is needed to deliver the retained I-frame of the requested TV channel to a client upon the arrival of the client's channel change request. Thus, each request will consume amount of system resources for a short period of time until the completion of the retained I-frame transmission. This is fundamentally different from the IP multicasting communication where the resource demands are directly determined by the number of multicasting channels. Furthermore, if we take the periodic burst feature of channel changing requests into account, the resource demands of the I-frame acceleration mechanism may become much larger than what the IPTV system can afford. Consequently, the scalability of the original I-frame acceleration mechanism can be severely degraded in practice. In particular, the scalability is mainly restricted by two factors, namely, the CCS's capacity (e.g., I/O capacity), as well as the bandwidth capacity of the links in the IPTV transmission network (e.g., the links between the CCS and clients). The demands regarding the above two aspects will be linearly increased as the number of concurrently served channel changing requests grows. In general, the CCS's capacity can be enhanced by employing more powerful servers, which is relatively easy compared to upgrading transmission networks (the second factor). The reason is clear that the transmission networks consist of a tremendous amount of links and intermediate nodes; most of the service providers cannot afford the costs of upgrading them (even in a medium scale). Therefore, it is highly desirable to propose efficient and easyimplementing schemes in order to optimize the scalability of the most widely used I-frame acceleration mechanism, particularly during commercial breaks of peak-hour durations.

\section{A Fairness-Based Admission Control (FAC) Scheme to Optimize the I-Frame Acceleration Mechanism}

In this section, aiming to optimize the scalability of the Iframe acceleration mechanism, we propose a novel admission control scheme by taking the fairness into account. The pros and cons of using this FAC scheme are also discussed.

4.1. General Assumptions. We investigate the IPTV service system as illustrated in Figure 2, where a CCS is installed as 
the entity to perform the I-frame acceleration mechanism based FCC methods. In addition, we assume the following.

(i) The service provider has reserved sufficient bandwidth in the delivery network for the concurrent multicasting of all the provided TV channels. If there are in total $K$ different TV channels provided and assume that all of them are constant bit-rate (CBR) or near CBR encoded, then the bandwidth reservation $B_{\text {multi }}$ on each link in the delivery path from the Video Source to every Access Node is fixed and is equal to the summation of all the channels' bandwidth demands. Particularly, $B_{\text {multi }}=\sum_{i=1}^{K} r_{\text {multi, } i}$, where $r_{\text {multi, } i}$ is the bit-rate for channel $i$. Note that $B_{\text {multi }}$ is irrelevant to the number of online clients but only the number of provided TV channels.

(ii) Upon receiving a channel change request from an IPTV client, the CCS immediately transmits a retained I-frame of the requested TV channel $i$ to the client by means of IP unicasting, with a CBR, which is generally higher than the channel's multicasting bitrate.

(iii) In order to deploy the I-frame acceleration mechanism based FCC methods, the service provider also reserves extra bandwidth on each link in the delivery path from the CCS to every Access Node to unicast the retained I-frames. However, due to resource limitations, the I-frame acceleration mechanism may run out of the reserved bandwidth on some bottleneck links. As a result, a portion of the unicasting flows carrying the retained I-frames for FCC may be blocked. Consequently, QoE degradation will be introduced to the clients downstream of the bottleneck link(s), especially during commercial breaks in peak-hours.

(iv) At a random instant of time, an online IPTV client is in one of the two modes, namely, zapping mode and viewing mode. In zapping mode a client continuously changes channels in a short time, while the viewing mode denotes a client keeping to watch a channel for a sufficiently long period of time (e.g., over 5 minutes).

(v) The number of channel change events generated by clients is correlated with the timeline of TV programs; that is, they burst during every commercial break with the interval of half an hour (cf. Figure 3).

4.2. A Fairness-Based Admission Control Scheme. In practice, an IPTV client may zap TV channels quite frequently during certain periods of time. We define this as the client's heavy zapping behaviour, which introduces a large amount of extra traffic loads to the transmission networks. The bandwidth reservation for deploying the FCC methods is limited in the transmission networks. Therefore, we believe it is not fair that the heavy zapping behaviour demands too much bandwidth resource and accordingly incurs negative influences on the clients who just occasionally zap channels. Taking this into consideration, we propose an optimization solution called fairness-based access control (FAC) scheme to restrict the heavy zapping behaviours of some clients and, consequently, to eliminate their negative impacts on the underlying transmission networks. The principle of this scheme can be depicted by the following three points.

Point 1. Monitor all the online IPTV clients by recording their recent channel changing histories.

Point 2. Based on Point 1, dynamically categorize a client into either the Heavy Zapper group or the Light Zapper group at instants this client changes channels.

Point 3. Taking the fairness into account, actively disable the unicasting of the retained I-frame to a client who is currently in the Heavy Zapper group.

More specifically, two logical modules are newly introduced into the IPTV service architecture as presented in Figure 2. They are Monitoring and Statistics Module (MSM) and Access Control Module (ACM), respectively. Due to the fact that both of them are logical modules, they can be installed close to or even be directly implemented on the CCS in practice.

MSM is adopted to conduct the following two tasks. First, for all the online clients, MSM monitors and calculates the frequency of the channel changing requests arriving at the CCS in real-time. Second, for each single client, MSM monitors its channel changing history by tracking the information of the client's most recent channel zapping events (excluding possible channel viewing events). More specifically, MSM continuously records and updates the zapping $d$ well time (i.e., the duration a client browses a channel in the zapping mode) for each of the online clients; for client $i$, the zapping dwell time corresponding to the most recent $N$ channel zapping events will be written into an array $Z_{i, j}(1 \leq j \leq N)$. Besides, MSM introduces a different weight $\alpha$ for the zapping dwell time connecting to the most recent channel zapping event (i.e., $Z_{i, 1}$ ), which is usually considered to be more important than the other elements in $Z_{i, j}$ to reflect the client's most recent behaviour and to predict the future trend. On that basis, the average zapping dwell time $T_{i}$ can be expressed by (1). In particular, three more detailed situations are presented in Figure 4. The first situation is demonstrated in Figure 4(a) where the client's past $N$ zapping events are continuous, and $T_{i}$ can be evaluated straightforward by (1). Figure 4(b) considers another situation with channel viewing event(s) amongst the $N$ zapping events. Thus, the view events should be eliminated when $T_{i}$ is evaluated. Figure 4 (c) illustrates the third situation with the client just turned on the TV, and currently the total number of zapping events $n$ is smaller than $N$. Thus, (2) is employed to replace (1) for the calculation of $T_{i}$ as follows:

$$
\begin{gathered}
T_{i}=\alpha \cdot Z_{i, 1}+(1-\alpha) \frac{\sum_{j=2}^{N} Z_{i, j}}{N-1}, \\
T_{i}=\frac{\sum_{j=1}^{n} Z_{i, j}}{n} .
\end{gathered}
$$

ACM is a logical entity to conduct the FAC scheme for all the I-frame unicasting flows served by the CCS. It is clear that each unicasting flow carries a retained I-frame of a requested 


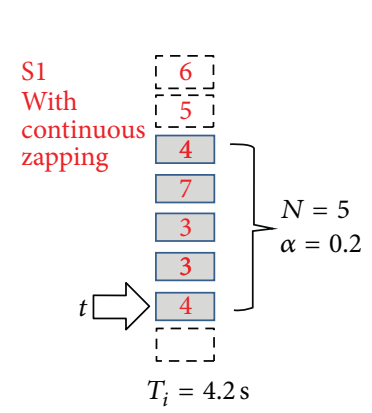

(a)

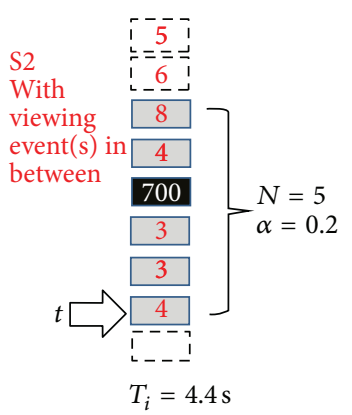

(b)

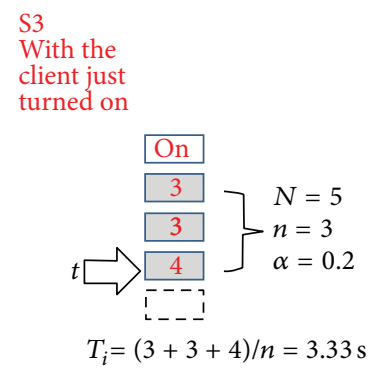

(c)

FIGURE 4: Three more detailed situations for applying the FAC scheme.

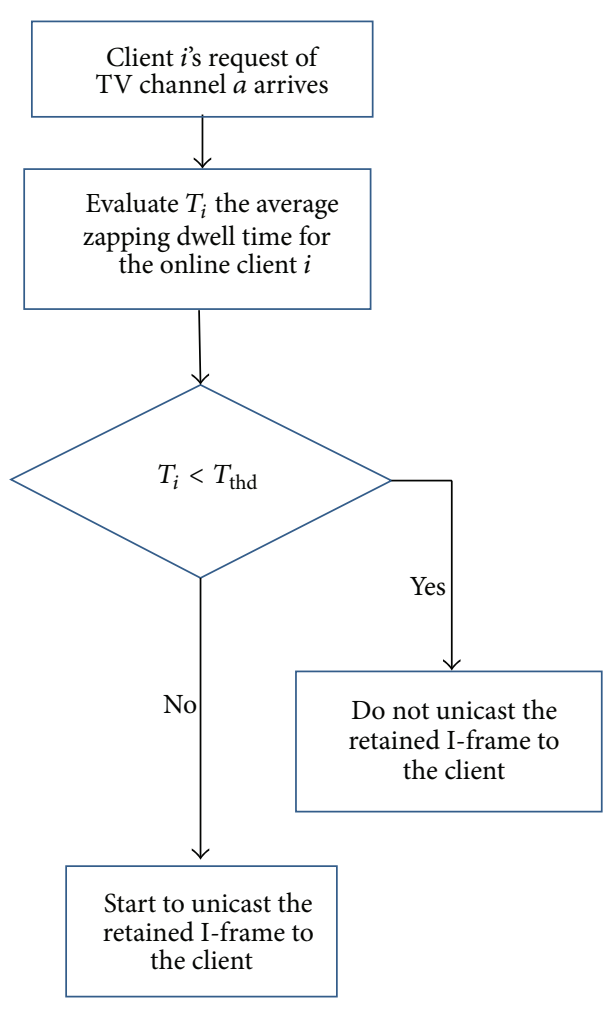

FIGURE 5: Flow chart to illustrate the FAC scheme.

TV channel with the aim to accelerate the channel changing process for the client requesting that channel. However, each unicasting flow consumes a considerable large amount of network bandwidth resources (although each unicasting flow may not last for a long time). When there is a burst of channel change requests arriving at the CCS, ACM is responsible for making sure that the limited network bandwidth resources can be used more fairly and efficiently, so that acceptable QoE can still be maintained for the clients. The implementation of our proposed FAC scheme can be demonstrated by the flow chart in Figure 5. As can be seen, the key step is to compare the evaluated $T_{i}$ and a threshold value $T_{\text {thd }}$, which is used to decide whether the current client is a heavy zapper or not.

Similar to $T_{i}$, the value of $T_{\text {thd }}$ is also dynamically determined and updated by the MSM. There are many different solutions to do this. One exemplary method may be time interval based. More precisely, after one fixed period of time, $T_{\text {thd }}$ should be recalculated according to the most recent updates of client requests arriving intensity. Another exemplary method could be based on the change of the client requests arriving intensity. In this case, if a currently updated requests intensity becomes significantly different than the mean value of several most recently evaluated intensities, either absolutely or relatively, $T_{\text {thd }}$ should be updated. The flow chart in Figure 6 demonstrates the process to determine and update $T_{\text {thd }}$ with algorithm $X$. Note that looking for a decent algorithm $X$ is outside of the scope of this paper and is left for further study. To summarise, Figure 7 demonstrates the functionalities and the relations between the two logical models (i.e., MSM and ACM).

4.3. Advantages and Disadvantages of the FAC Scheme. The advantage of using our FAC scheme is obvious. With the FAC scheme, the network bandwidth demands by the Iframe acceleration mechanism can be decreased; the CBP on the CCS due to bandwidth limitation can also be reduced. Accordingly, the scalability of the original I-frame acceleration mechanism can be effectively optimized.

On the other hand, the above advantages are not achieved without cost. Our FAC scheme sacrifices the profits of certain clients who are currently conducting heavy zapping (i.e., in the Heavy Zapper group), by means of actively and intelligently blocking the retained I-frame transmissions for FCC. However, we believe that this punishment for the heavy zapping behaviour preserves the profits for the clients in the light zapper group. As a result, the I-frame acceleration mechanism can treat different clients more fairly with adopting this proposed FAC scheme.

\section{Performance Evaluation by Means of Simulation}

In this section, the performance of our proposed FAC scheme is evaluated by means of simulation. In particular, we present a case study concerning a typical commercial break in peakhours.

5.1. Simulation Introduction. We focus on a single bottleneck link $L N$ between the CCS and a certain Access Node and 


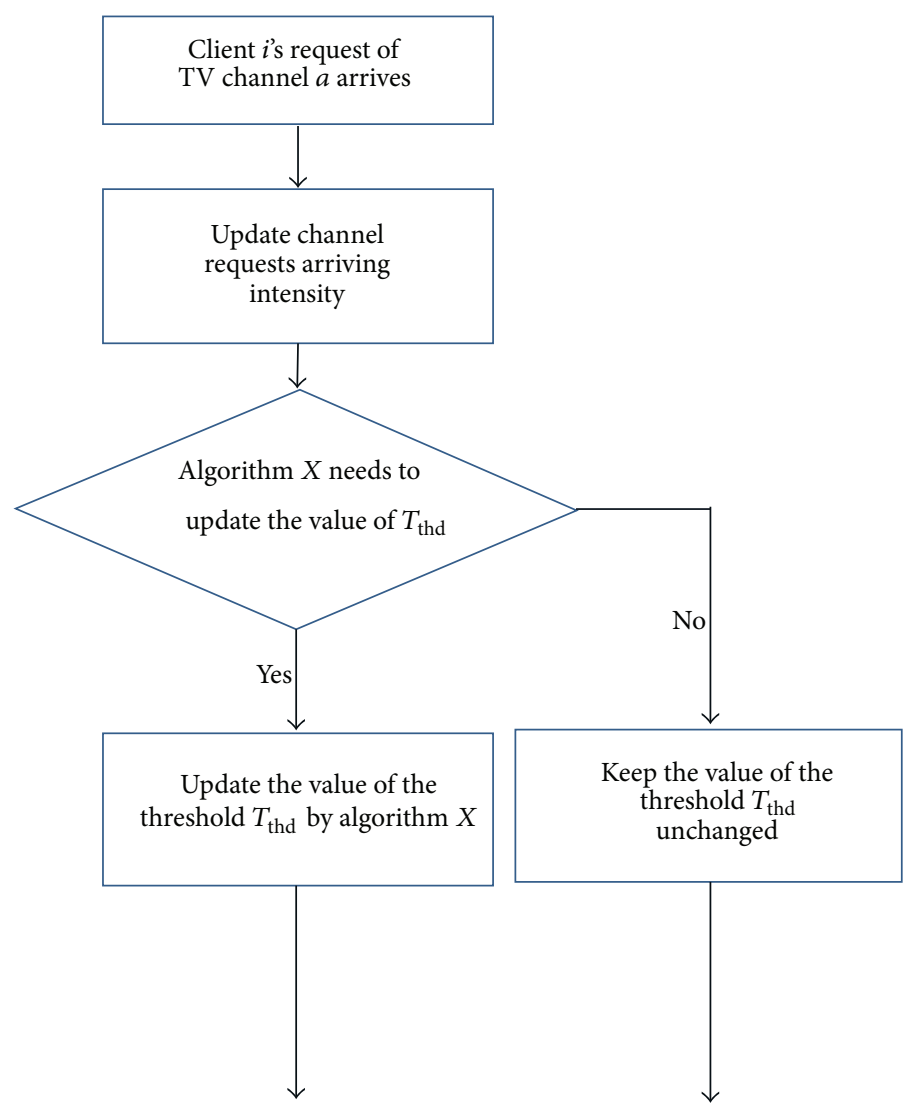

FIgURE 6: Flow chart to show the determination of $T_{\text {thrd }}$.

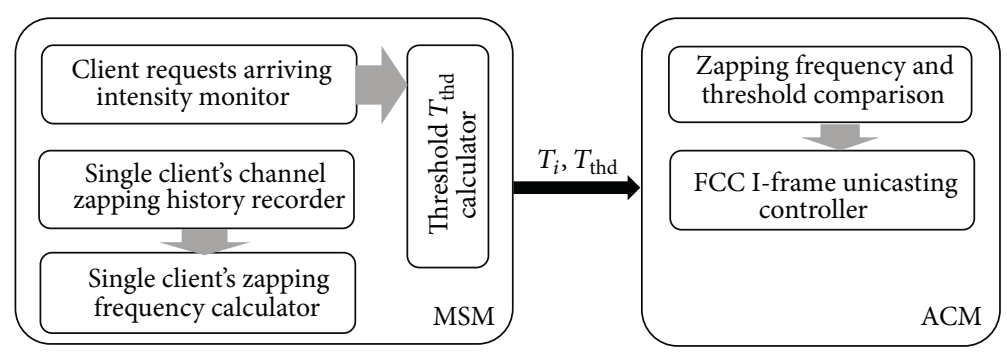

FIGURE 7: The functionalities and the relations between the MSM and the ACM modules.

assume that there is no other bottleneck links in the channel delivery path. On the link $L N$, two different performance metrics, that is, the real-time bandwidth demand and the CBP for FCC introduced by the I-frame acceleration mechanism; are evaluated. Here the second metrics are defined as the probability of blocking the retained I-frame unicasting streams due to resource limitation. To this end, we have developed a trace-driven simulation model based on the $n$ dimensional state vector below:

$$
\text { (UserBW_1, UserBW_2,.., UserBW_n), }
$$

where $n$ is the total number of clients downstream of the link $L N$. The vector is used for recording the current status of the clients; that is, the $i$ th element UserBW $i$ in the vector represents the current bandwidth requirement introduced by
FCC for client $i$. Note that UserBW $\_i$ should be 0 except in the duration when the CCS is expected to deliver a retained I-frame to client $i$. Thus, the summation of all the elements is the aggregate bandwidth demand on $L N$ for all the downstream clients. The simulation consists of the following four steps.

Step 1. Generate a traffic trace for each of the clients downstream of the link $L N$, which should include channel request arriving events, as well as FCC completion events (i.e., the finish of transmitting a retained I-frame). Besides, the length of each single trace should be larger than or at least equal to $T_{\mathrm{cb}}$, the time duration will be simulated.

Step 2. Derive an aggregate trace for all the clients by combining all the single traces generated in Step 1. 
Step 3. Start to simulate client channel changing events as well as FCC completion events according to the aggregate trace. During the simulation, the bandwidth requirement on the link $L N$ for I-frame accelerations is monitored and recorded in real-time.

Step 4. At the end of the simulation, CBPs for FCC in both the entire simulation duration $T_{\mathrm{cb}}$ and in a certain portion of it are evaluated based on the recorded call blocking events and the total number of channel change requests during the simulation.

We need to study two different types of situations, namely, the situations with and without using our proposed FAC scheme, with the aim to demonstrate that to what extent our FAC scheme can improve the scalability of the original Iframe acceleration mechanism.

During the simulation, the transmission of a retained Iframe (for FCC) may be blocked either due to bandwidth resource constraints or caused by using the proposed FAC scheme. In that case, the channel changing acceleration for a specific client request is failed, and the client has to wait for extra time to get the first I-frame (in the multicasting stream of the requested channel) to be displayed on the screen. We call this extra time directly introduced by the FAC scheme as FCC failure delay (FFD). The Average FFD is monitored as the third performance metric, which is defined as the sum of FFDs normalized by the total number of channel requests in the simulated time slot.

5.2. Case Study: Application of the FAC Scheme in a Typical Commercial Break in Peak-Hours. Based on the above simulation model, we conduct performance evaluations for the FAC scheme when it is applied to a typical commercial break (with length $T_{\mathrm{cb}}$ ) in peak-hours. When a large number of channel requests burst, the heavy traffic load introduced by using the I-frame acceleration mechanism based FCC methods can strongly impact on IPTV systems. Below are some additional assumptions for this case study.

(i) The system provides both SDTV and HDTV channels, both of which are CBR encoded with the multicasting transmission bit-rates $R_{\text {Multi,SD }}$ and $R_{\text {Multi,HD}}$, respectively.

(ii) When a client conducts a channel change for an SDTV (or HDTV) channel, the CCS promptly unicasts a retained I-frame of the requested channel to the client with CBR $R_{\mathrm{Uni}, \mathrm{SD}}$ (or $R_{\mathrm{Uni}, \mathrm{HD}}$ ), which is generally larger than the channel's multicasting bit-rate $R_{\text {Multi,SD }}$ and satisfies (4) (or (5) for HDTV channel). Consider

$$
\begin{array}{cc}
R_{\mathrm{Uni}, \mathrm{SD}}=\alpha_{\mathrm{SD}} \cdot R_{\mathrm{Multi}, \mathrm{SD}} & \left(\alpha_{\mathrm{SD}}>1\right), \\
R_{\mathrm{Uni}, \mathrm{HD}}=\alpha_{\mathrm{HD}} \cdot R_{\mathrm{Multi}, \mathrm{HD}} & \left(\alpha_{\mathrm{HD}}>1\right) .
\end{array}
$$

(iii) The bandwidth reservation on the bottleneck link $L N$ for unicasting the retained I-frames is fixed as $B W$ (in practical IPTV systems, $B W$ should be determined by the service providers with considering other factors, such as the average offered traffic).

(iv) The number of IPTV clients downstream of the link $L N$ is fixed as $N_{\text {client }}$ during the considered commercial break, which means that there is no client entering into or leaving from the system. The clients can choose both SDTV and HDTV channels (i.e., with the probability $\beta$ to select SDTV channels). In addition, these clients behave independently.

(v) Each of the clients begins to zap TV channels once the commercial break starts. A client continues to zap channels for $L$ times. $L$ conforms to a truncated Poisson distribution with the mean value $\bar{L}$, the maximum value $L_{\mathrm{MAX}}$, and the minimum value $L_{\mathrm{MIN}}$. After each round of channel zapping, a client enters into view model (i.e., watches a channel for a relatively long time). Thus, the probability mass function (PMF) of $L$ can be expressed by (6), where $L_{\mathrm{MIN}} \leq k \leq L_{\mathrm{MAX}}$ should be satisfied. Consider

$$
f(k ; \bar{L})=\operatorname{Pr}(L=k)=\frac{\left(\bar{L}^{k} \cdot e^{-\bar{L}}\right) / k !}{\sum_{j=L_{\mathrm{MIN}}}^{L_{\mathrm{MAX}}}\left(\left(\bar{L}^{j} \cdot e^{-\bar{L}}\right) / j !\right)} .
$$

(vi) For each single client, the zapping dwell time $T_{\mathrm{zdt}}$ (denoting the duration a client browses a certain channel in the zapping mode) conforms to a truncated negative Exponential distribution with the average value $\bar{T}_{\mathrm{zdt}}$, the maximum value $T_{\mathrm{zdt}, \mathrm{MAX}}$, and the minimum value $T_{\mathrm{zdt}, \mathrm{MIN}}$. Thus, the probability density function (PDF) of $T_{\mathrm{zdt}}$ can be expressed by (7), where $T_{\mathrm{zdt}, \mathrm{MIN}} \leq x \leq T_{\mathrm{zdt}, \mathrm{MAX}}$ should be satisfied

$$
f\left(x ; \frac{1}{\bar{T}_{\mathrm{zdt}}}\right)=\frac{\bar{T}_{\mathrm{zdt}} \cdot e^{-\bar{T}_{\mathrm{zdt}} \cdot x}}{\int_{T_{\mathrm{zd}, \mathrm{MIN}}}^{T_{\mathrm{zdt}, \mathrm{MAx}}}\left(\bar{T}_{\mathrm{zdt}} \cdot e^{-\bar{T}_{\mathrm{zdt}} \cdot x}\right)} .
$$

(vii) Assume that the size of the retained I-frames conforms to a truncated Normal distribution. More specifically, for an SDTV channel the mean value, the maximum value, and the minimum value of the I-frame size $S_{\mathrm{SD}}$ are $\bar{S}_{\mathrm{SD}}, S_{\mathrm{SD}, \mathrm{MAX}}$, and $S_{\mathrm{SD}, \mathrm{MIN}}$, respectively. The standard deviation of $S_{\mathrm{SD}}$ is $\sigma_{\mathrm{sd}}$. On the other hand, for an HDTV channel the mean value, the maximum value, and the minimum value of the I-frame size $S_{\mathrm{HD}}$ is $\bar{S}_{\mathrm{HD}}, S_{\mathrm{HD}, \mathrm{MAX}}$ and $S_{\mathrm{HD}, \mathrm{MIN}}$, respectively. The standard deviation of $S_{\mathrm{HD}}$ is $\sigma_{\mathrm{hd}}$. Thus, the PDFs of $S_{\mathrm{SD}}$ and $S_{\mathrm{HD}}$ can be expressed by (8) and (9), respectively. Besides, the inequalities $S_{\mathrm{SD}, \mathrm{MIN}} \leq x \leq S_{\mathrm{SD}, \mathrm{MAX}}$, and $S_{\mathrm{HD}, \mathrm{MIN}} \leq y \leq S_{\mathrm{HD}, \mathrm{MAX}}$ should be respected. Consider

$$
\begin{gathered}
f(x)=\frac{\left(1 / \sqrt{2 \pi \sigma_{\mathrm{sd}}^{2}}\right) \cdot e^{-\left(\left(x-\bar{S}_{\mathrm{SD}}\right)^{2} / 2 \sigma_{\mathrm{sd}}^{2}\right)}}{\int_{S_{\mathrm{SD}, \mathrm{MIN}}}^{S_{\mathrm{SD}, \mathrm{N}}}\left[\left(1 / \sqrt{2 \pi \sigma_{\mathrm{sd}}^{2}}\right) \cdot e^{-\left(\left(x-\bar{S}_{\mathrm{SD}}\right)^{2} / 2 \sigma_{\mathrm{sd}}^{2}\right)}\right]}, \\
f(y)=\frac{\left(1 / \sqrt{2 \pi \sigma_{\mathrm{sd}}^{2}}\right) \cdot e^{-\left(\left(y-\bar{S}_{\mathrm{HD}}\right)^{2} /\left(2 \sigma_{\mathrm{hd}}^{2}\right)\right)}}{\int_{S_{\mathrm{HD}, \mathrm{MIN}}}^{S_{\mathrm{HD}, \mathrm{MAX}}}\left[\left(1 / \sqrt{2 \pi \sigma_{\mathrm{hd}}^{2}}\right) \cdot e^{-\left(\left(y-\bar{S}_{\mathrm{HD}}\right)^{2} / 2 \sigma_{\mathrm{hd}}^{2}\right)}\right]} .
\end{gathered}
$$


TABLE 2: Parameter values for the case study.

\begin{tabular}{|c|c|c|}
\hline Notation & Description & Values \\
\hline$T_{\mathrm{cb}}$ & Length of the considered commercial break & 5 Minutes \\
\hline$B W$ & $\begin{array}{l}\text { Bandwidth reserved for unicasting the retained I-frames for FCC on the } \\
\text { bottleneck link } L N\end{array}$ & $500 \mathrm{Mbps}$ \\
\hline$N_{\text {client }}$ & The number of IPTV clients downstream of the link $L N$ & 4000 \\
\hline$\beta$ & $\begin{array}{l}\text { The probability a client chooses an SDTV channel when conducting the } \\
\text { channel changing }\end{array}$ & 0.7 \\
\hline$R_{\text {Multi,SD }}$ & CBR of multicasting an SDTV channel & $4 \mathrm{Mbps}$ \\
\hline$R_{\text {Multi,HD }}$ & CBR of multicasting an HDTV channel & $8 \mathrm{Mbps}$ \\
\hline$\alpha_{\mathrm{SD}}$ & I-frame acceleration ratio for an SDTV channel & 1.2 \\
\hline$\alpha_{\mathrm{HD}}$ & I-frame acceleration ratio for an HDTV channel & 1.4 \\
\hline$R_{\text {Uni,SD }}$ & CBR of unicasting the retained I-frame for SDTV channels & $4.8 \mathrm{Mbps}\left(=\alpha_{\mathrm{SD}} \cdot R_{\mathrm{Multi}, \mathrm{SD}}\right)$ \\
\hline$R_{\text {Uni,HD }}$ & CBR of unicasting the retained I-frame for HDTV channels & $11.2 \operatorname{Mbps}\left(=\alpha_{\mathrm{HD}} \cdot R_{\mathrm{Multi}, \mathrm{HD}}\right)$ \\
\hline$L$ & $\begin{array}{l}\text { Number of zapping times in the commercial break; conforming to a truncated } \\
\text { Poisson distribution ( } \bar{L} \text { is the mean, } L_{\mathrm{MAX}} \text { is the upper bound, and } L_{\mathrm{MIN}} \text { is } \\
\text { the lower bound) }\end{array}$ & $\bar{L}=15 ; L_{\mathrm{MAX}}=100 ; L_{\mathrm{MIN}}=5$ \\
\hline$T_{\mathrm{zdt}}$ & $\begin{array}{l}\text { Zapping dwell time (i.e., the duration a client browses a certain channel in the } \\
\text { zapping mode) conforms to a truncated negative Exponential distribution } \\
\text { ( } \bar{T}_{\mathrm{zdt}} \text { is the mean, } T_{\mathrm{zdt}, \mathrm{MAX}} \text { is the upper bound, and } T_{\mathrm{zdt}, \mathrm{MIN}} \text { is the lower bound) }\end{array}$ & $\begin{aligned} \bar{T}_{\mathrm{zdt}}= & 10 \mathrm{~s} ; T_{\mathrm{zdt}, \mathrm{MAX}}=20 \mathrm{~s} \\
& T_{\mathrm{zdt}, \mathrm{MIN}}=1.5 \mathrm{~s}\end{aligned}$ \\
\hline$S_{\mathrm{SD}}$ & $\begin{array}{l}\text { Size of an I-frame in an SDTV channel, which conforms to truncated Normal } \\
\text { distributions }\left(\bar{S}_{\mathrm{SD}} \text { is the mean, } S_{\mathrm{SD}, \mathrm{MAX}} \text { is the upper bound, } S_{\mathrm{SD}, \mathrm{MIN}} \text { is the }\right. \\
\text { lower bound, and } \sigma_{\mathrm{sd}} \text { is the standard deviation) }\end{array}$ & 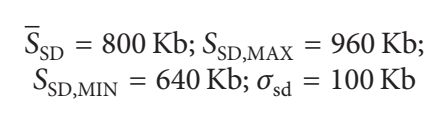 \\
\hline$S_{\mathrm{HD}}$ & $\begin{array}{l}\text { Size of an I-frame in an HDTV channel, which conforms to truncated } \\
\text { Normal distributions }\left(\bar{S}_{\mathrm{HD}} \text { is the mean, } S_{\mathrm{HD}, \mathrm{MAX}} \text { is the upper bound, } S_{\mathrm{HD}, \mathrm{MIN}}\right. \\
\text { is the lower bound, and } \sigma_{\mathrm{hd}} \text { is the standard deviation) }\end{array}$ & $\begin{array}{c}\bar{S}_{\mathrm{HD}}=1.6 \mathrm{Mb} ; S_{\mathrm{HD}, \mathrm{MAX}}=1.92 \mathrm{Mb} \\
S_{\mathrm{HD}, \mathrm{MIN}}=1.28 \mathrm{Mb} ; \sigma_{\mathrm{hd}}=0.2 \mathrm{Mb}\end{array}$ \\
\hline$T_{\text {Iframe,SD }}$ & Time needed to transmit a retained I-frame of an SDTV channel & $T_{\text {Iframe,SD }}=\frac{S_{\mathrm{SD}}}{R_{\text {Uni,SD }}}$ \\
\hline$T_{\text {Iframe,HD }}$ & Time needed to transmit a retained I-frame of an HDTV channel & $T_{\text {Iframe,HD }}=\frac{S_{\mathrm{HD}}}{R_{\mathrm{Uni}, \mathrm{HD}}}$ \\
\hline$D_{\mathrm{SD}}$ & $\begin{array}{l}\text { FCC failure delay (FFD) for an SDTV channel; subjects to a truncated } \\
\text { negative Exponential distribution }\left(\bar{D}_{\mathrm{SD}} \text { is the mean, } D_{\mathrm{SD}, \mathrm{MAX}} \text { is the upper }\right. \\
\left.\text { bound, and } D_{\mathrm{SD}, \mathrm{MIN}} \text { is the lower bound }\right)\end{array}$ & $\begin{aligned} \bar{D}_{\mathrm{SD}}= & 0.6 \mathrm{~s} ; D_{\mathrm{SD}, \mathrm{MIN}}=0.2 \mathrm{~s} \\
& D_{\mathrm{SD}, \mathrm{MAX}}=1 \mathrm{~s}\end{aligned}$ \\
\hline$D_{\mathrm{HD}}$ & $\begin{array}{l}\text { FCC failure delay (FFD) for an HDTV channel; subjects to a truncated } \\
\text { negative Exponential distribution }\left(\bar{D}_{\mathrm{HD}} \text { is the mean, } D_{\mathrm{HD}, \mathrm{MAX}} \text { is the upper }\right. \\
\text { bound, and } D_{\mathrm{HD}, \mathrm{MIN}} \text { is the lower bound) }\end{array}$ & $\begin{array}{c}\bar{D}_{\mathrm{HD}}=0.8 \mathrm{~s} ; D_{\mathrm{HD}, \mathrm{MIN}}=0.3 \mathrm{~s} \\
D_{\mathrm{HD}, \mathrm{MAX}}=1.3 \mathrm{~s}\end{array}$ \\
\hline
\end{tabular}

(viii) The time needed to transmit the retained I-frame is denoted as $T_{\text {I-frame,SD }}$ and $T_{\text {I-frame,HD }}$ for the SDTV and the HDTV channels, respectively. $T_{\mathrm{I} \text {-frame,SD }}$ and $T_{\text {I-frame,HD }}$ can be directly determined by the size of the I-frame and the unicasting bit-rate. Consequently, (10) should be fulfilled as follows:

$$
\begin{aligned}
T_{\text {I-frame,SD }} & =\frac{S_{\mathrm{SD}}}{R_{\mathrm{Uni}, \mathrm{SD}}}, \\
T_{\text {I-frame } \mathrm{HD}} & =\frac{S_{\mathrm{HD}}}{R_{\mathrm{Uni}, \mathrm{HD}}} .
\end{aligned}
$$

(ix) As discussed in the previous subsection, FFD is defined as the extra channel changing latency brought by the FAC scheme. A set of factors including those listed in Table 1 can contribute to FFD. Therefore, it is reasonable to assume that FFD subjects to a truncated negative Exponential distribution. For the SDTV and HDTV channels, we denote FFD as $D_{\mathrm{SD}}$ and $D_{\mathrm{HD}}$, respectively.
Table 2 lists the parameter values we used for this case study. In the simulation experiments, we have applied our FAC scheme with setting the parameters $N=3, \alpha=$ 0.5 (cf. Section 4.2). Besides, three different values for the threshold $T_{\text {thd }}$ are tested. The simulation results are plotted in Figures 8-11. More specifically, Figures 8, 9, and 10 present the bandwidth demand variations during the simulated commercial break for the three different values of $T_{\text {thd }}$, respectively. In each of these figures, the upper curve represents the situation with only using the original I-frame acceleration mechanism. As can be seen, the aggregate bandwidth demand increases dynamically once the simulation starts. As time goes, it reaches a relatively stable status; namely, the bandwidth demand vibrates around a fixed value. After that, the bandwidth demand starts to decrease slowly and gradually gets close to zero at the end of the commercial break. The other curve located underneath is for the situation of using our FAC scheme with a specific value of $T_{\text {thd }}$. The lower curve illustrates similar features as the upper one. However, it demands much less bandwidth (e.g., up to $50 \%$ percent less in Figure 10) compared to the situation when FAC scheme is 


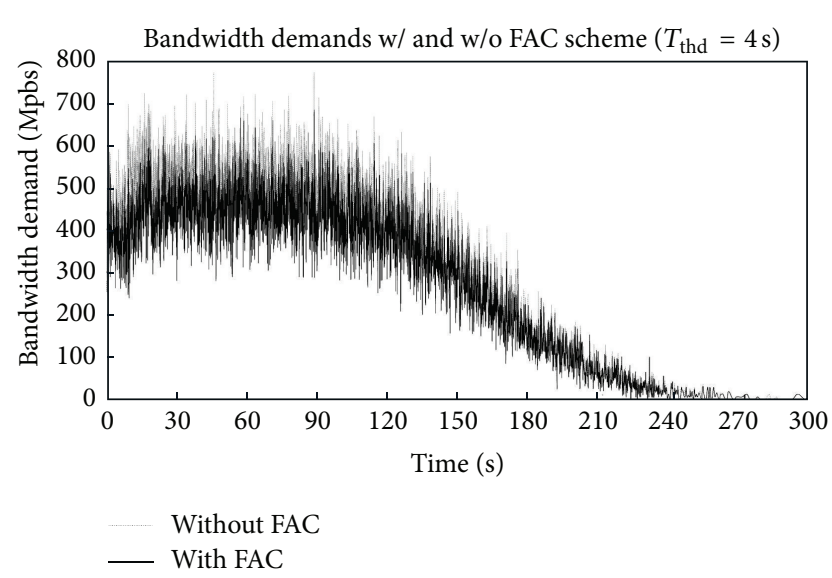

Figure 8: Bandwidth demand on link $L N$ for transmitting the retained I-frames $\mathrm{w} /$ and w/o using the FAC scheme $\left(T_{\text {thd }}=\right.$ 4 seconds).

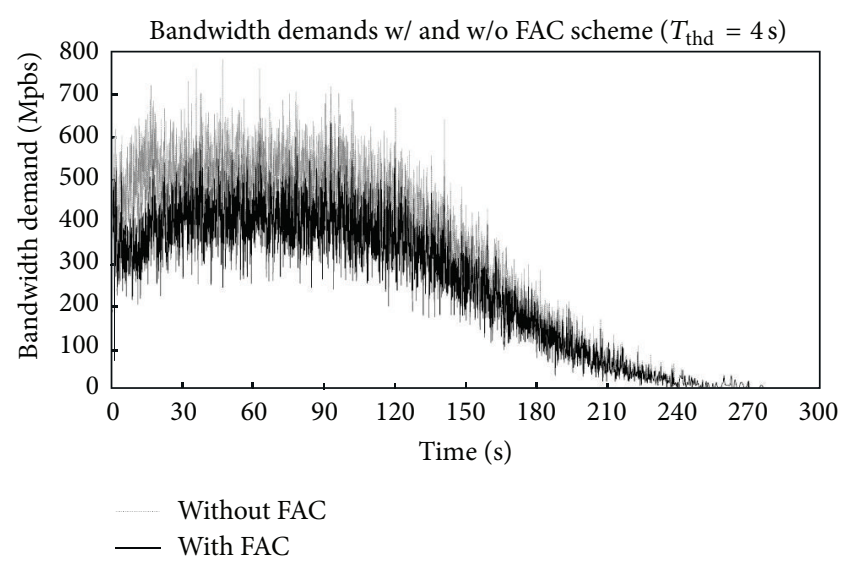

FIgure 9: Bandwidth demand on link $L N$ for transmitting the retained I-frames $\mathrm{w} /$ and w/o using the FAC scheme $\left(T_{\text {thd }}=\right.$ 5 seconds).

not applied. In addition, when comparing all the lower curves in the three figures, we observed that our FAC scheme with a larger value of $T_{\text {thd }}$ can introduce a more significant reduction of bandwidth demands. In Figure 11, we present the results of the CBPs for FCC evaluated for the intervals of every 30 seconds (in this case, we get $10(=5 \mathrm{~min} / 30 \mathrm{~s}$ ) sampling points on each curve) in the simulated commercial break. There are in total four curves presented, including the uppermost one for the situation without using the FAC scheme and the three lower ones using the FAC scheme but corresponding to the three different values of $T_{\text {thd }}$. We can observe that the larger the $T_{\text {thd }}$ is, the lower the corresponding curve locates, that is, the smaller the CBPs for FCC are.

The four figures above illustrate the benefits of using our FAC scheme. Those gains are achieved by riding the cost of increasing channel changing delay for the heavy zappers. It is agreed that a thorough investigation of the cost is indispensable for a complete study. We therefore evaluate the Average FFD in a scenario where limited bandwidth

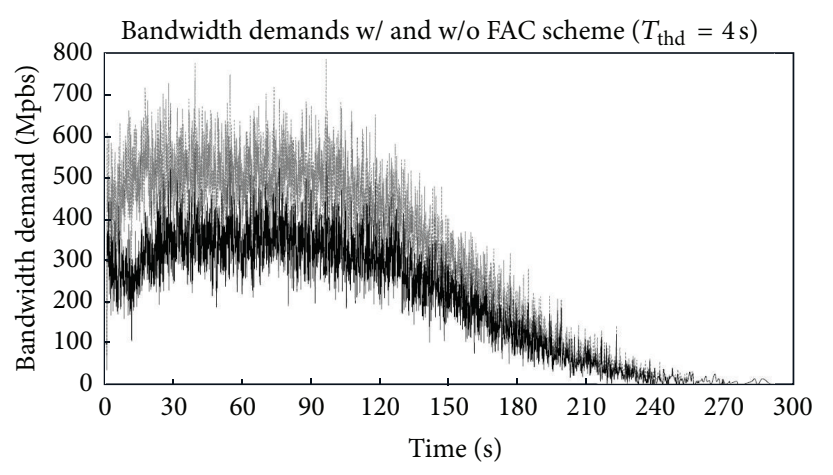

Without FAC

— With FAC

Figure 10: Bandwidth demand on link $L N$ for transmitting the retained I-frames $\mathrm{w} /$ and $\mathrm{w} / \mathrm{o}$ using the FAC scheme $\left(T_{\text {thd }}=\right.$ 6 seconds).

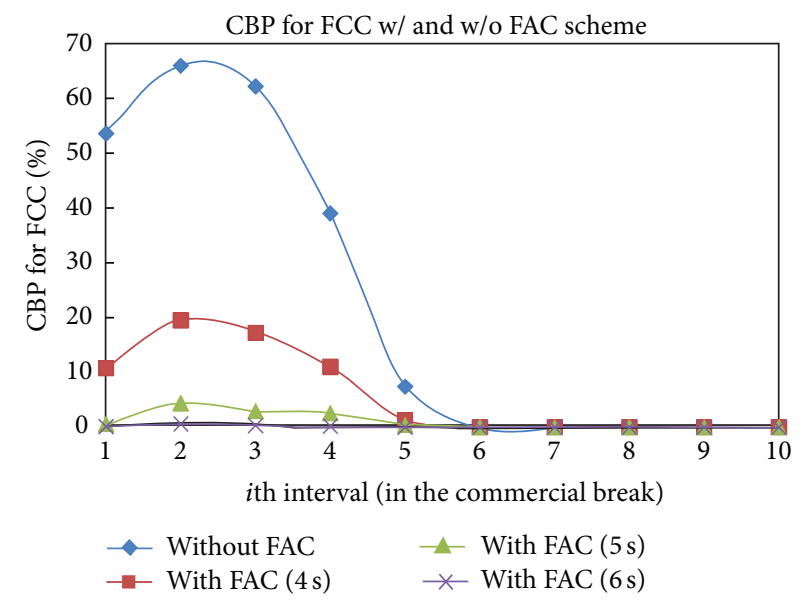

FIGURE 11: FCC CBP evaluated on link $L N$ w/ and w/o applying the FAC scheme for different values of $T_{\text {thd }}$.

reservation is assumed on the bottleneck link $L N$ (i.e., $B W=$ $500 \mathrm{Mbps}$ ). This indicates that an FCC failure event can be either attributed to the bandwidth limits or incurred by using the FAC scheme. The simulation results for the three different values of $T_{\text {thd }}$ are plotted on Figure 12. As can be seen, the average FFD linearly increases as $T_{\text {thd }}$ grows. The general trend of each curve is to slowly decrease as the time goes in the simulation duration. The maximum value is around 0.27 second in the curve $T_{\text {thd }}=6 \mathrm{~s}$. Since it appears just at the beginning of the commercial break when increasingly more clients start to zap channels, we believe it is acceptable as an average FFD value. And we also note that for heavy zappers the situation would be worse, while for clients not addicted to channel zapping, the average delay would be much shorter.

To summarise, the simulation results imply that our FAC scheme can effectively optimize the scalability of the original I-frame acceleration mechanism based FCC methods by dramatically decreasing the bandwidth demands. In particular, if the bandwidth reservation for transmitting the retained Iframes is limited in the delivery network, the FAC scheme 


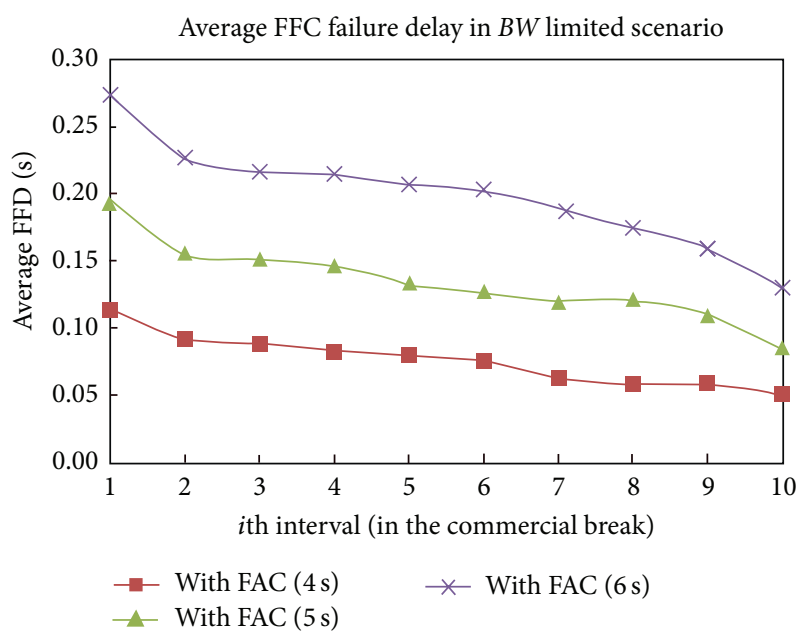

FIGURE 12: Average FFC failure delay (FFD) for different values of $T_{\text {thd }}$ in bandwidth limited scenarios $(B W=500 \mathrm{Mbps})$.

is capable of significantly reducing the CBPs for FCC, with only slightly increasing the average channel changing delay (i.e., average FFD). It is also observed that, when the reserved bandwidth is fixed, our proposed FAC scheme performs better in terms of reducing bandwidth requirements and CBP for larger values of $T_{\text {thd }}$. It is however not true for the average FFD, which just gets worse as $T_{\text {thd }}$ increases. In addition, we emphasis that, as an important scalability optimization method for the original I-frame acceleration mechanism, the FAC scheme is easy to be implemented by IPTV service providers in reality; the performance of the FAC scheme is closely related to both the bandwidth reservation for the I-frame acceleration mechanism, as well as the value of $T_{\text {thd }}$. Therefore, IPTV service providers should carefully choose the values for these parameters based on their practical needs.

\section{Summary and Outlook}

In this paper, we focused on optimizing the scalability of the I-frame acceleration mechanism, which is currently most widely used in IPTV systems to decrease channel change delay caused by I-frame acquisition. Existing literature indicates that channel changing events in an IPTV system are temporally correlated and tend to be periodically bursting (cf. Figure 3). Due to this phenomenon, applying the Iframe acceleration mechanism may introduce a large amount of extra traffic loads which can run out of the available resources (in particular, the limited network bandwidth) in IPTV systems. Accordingly, the scalability of the I-frame acceleration mechanism can be largely degraded, especially during commercial breaks in peak-hours. Aiming to solve this problem (i.e., optimize the scalability), we proposed an easy-implementing fairness-based admission control (FAC) scheme, as a scalability optimization method for the original I-frame acceleration mechanism. The performance of the FAC scheme is evaluated by comprehensive simulation experiments. The simulation results show that our proposed FAC scheme has the ability to significantly decrease the bandwidth demand as well as the CBP for FCC. Hence it can optimize the scalability of the I-frame acceleration mechanism, with only slightly increasing the average channel changing delay by temporarily disabling FCC for the clients who are addicted to frequent channel zapping.

Our planned work in the future will be the derivation of an optimized algorithm to dynamically determine the threshold value $T_{\text {thd }}$, with the purpose to further improve the performance of the FAC scheme. On the other hand, more realistic user behaviour models in terms of channel changing events during commercial breaks are desired and should be proposed for the performance evaluation for the FAC scheme, when more practical measurement data become available for us.

\section{Conflict of Interests}

The authors declare that there is no conflict of interests regarding the publication of this paper.

\section{References}

[1] H. Uzunalioglu, "Channel change delay in IPTV systems," in Proceedings of the 6th IEEE Consumer Communications and Networking Conference (CCNC '09), Las Vegas, Nev, USA, January 2009.

[2] C. Cho, I. Han, Y. Jun, and H. Lee, "Improvement of channel zapping time in IPTV services using the adjacent groups joinleave method," in Proceedings of the 6th International Conference on Advanced Communication Technology, vol. 2, pp. 971-975, February 2004.

[3] Lucent Technologies, "Optimization of IPTV multicast traffic transport over next generation metro networks," in Proceedings of the 12th International Telecommunications Network Strategy and Planning Symposium, November 2006.

[4] “RFC 4607: Source-Specific Multicast for IP”, August 2006.

[5] J. M. Boyce and A. M. Tourapis, "Fast efficient channel change," in Proceedings of the nternational Conference on Consumer Electronics, January 2005.

[6] “Fast Channel Changing in RTP," ISMA Technical White Paper, 2007.

[7] H. Schwarz, D. Marpe, and T. Wiegand, "Overview of the scalable video coding extension of the H.264/AVC standard," IEEE Transactions on Circuits and Systems for Video Technology, vol. 17, no. 9, pp. 1103-1120, 2007.

[8] P. T. Barrett, J. A. Baldwin, and D. L. Green, "Fast channel change," US 7562375 B2, 2009.

[9] D. Banodkar, K. K. Ramakrishnan, S. Kalyanaraman, A. Gerber, and O. Spatscheck, "Multicast instant channel change in IPTV systems," in Proceedings of the 3rd IEEE/Create-Net International Conference on Communication System Software and Middleware, pp. 370-379, January 2008.

[10] T. Qiu, Z. Ge, S. Lee, J. Wang, J. Xu, and Q. Zhao, "Modeling user activities in a large IPTV system," in Proceedings of the 9th ACM SIGCOMM Conference on Internet Measurement (IMC '09), pp. 430-441, Chicago, Ill, USA, 2009.

[11] F. Ramos, F. Song, P. Rodriguez, R. Gibbens, J. Crowcroft, and I. H. White, "Constructing an IPTV workload model," in Proceedings of the ACM SIGCOMM Conference on Data Communication (SIGCOMM '09), poster session, Barcelona, Spain, 2009. 
[12] G. Yu, T. Westholm, M. Kihl et al., "Analysis and characterization of IPTV user behavior," in Proceedings of the IEEE International Symposium on Broadband Multimedia Systems and Broadcasting, BMSB 2009, Bilbao, Spain, May 2009.

[13] M. Cha, P. Rodriguez, J. Growcroft, S. Moon, and X. Amatriain, "Watching television over an IP network," in Proceedings of the 8th ACM SIGCOMM Conference on Internet Measurement (IMC '08), pp. 71-84, Vouliagmeni, Greece, 2008.

[14] A. Abdollahpouri, B. E. Wolfinger, J. Lai, and C. Vinti, "Elaboration and formal description of IPTV user models and their application to IPTV system analysis," in Proceedings of the GI/ITG Workshop on Measurement, Modelling and Evaluation of Computing System (MMBnet '11), pp. 67-79, Hamburg, Germany, 2011.

[15] A. Abdollahpouri, B. E. Wolfinger, J. Lai, and C. Vinti, "Elaboration and formal description of IPTV user modeling the behavior of IPTV users with application to call blocking probability analysis," Praxis der Informationsverarbeitung und Kommunikation, vol. 35, pp. 75-81, 2012. 


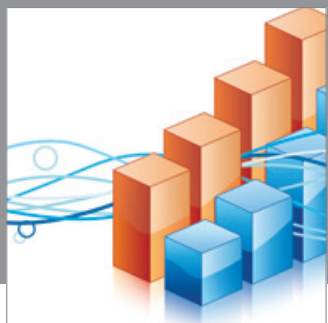

Advances in

Operations Research

mansans

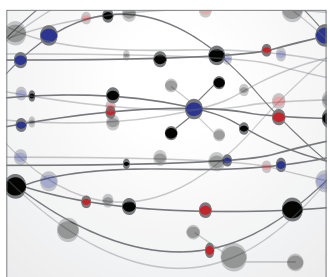

The Scientific World Journal
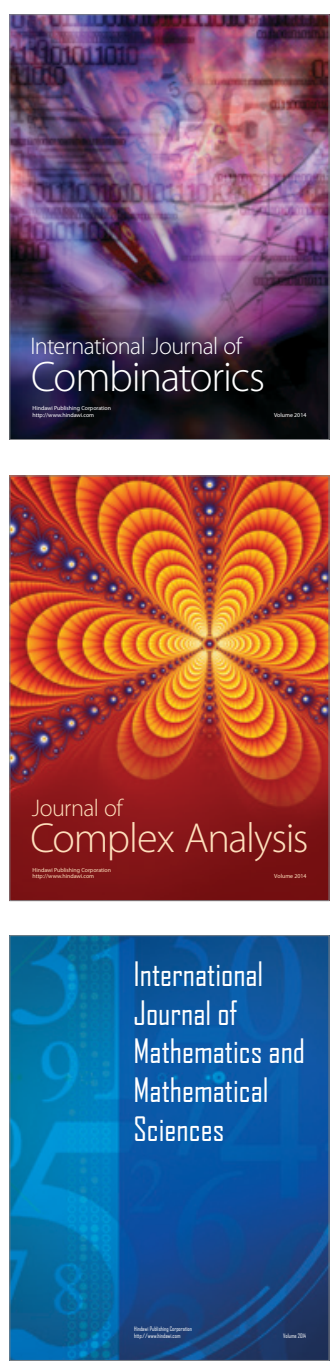
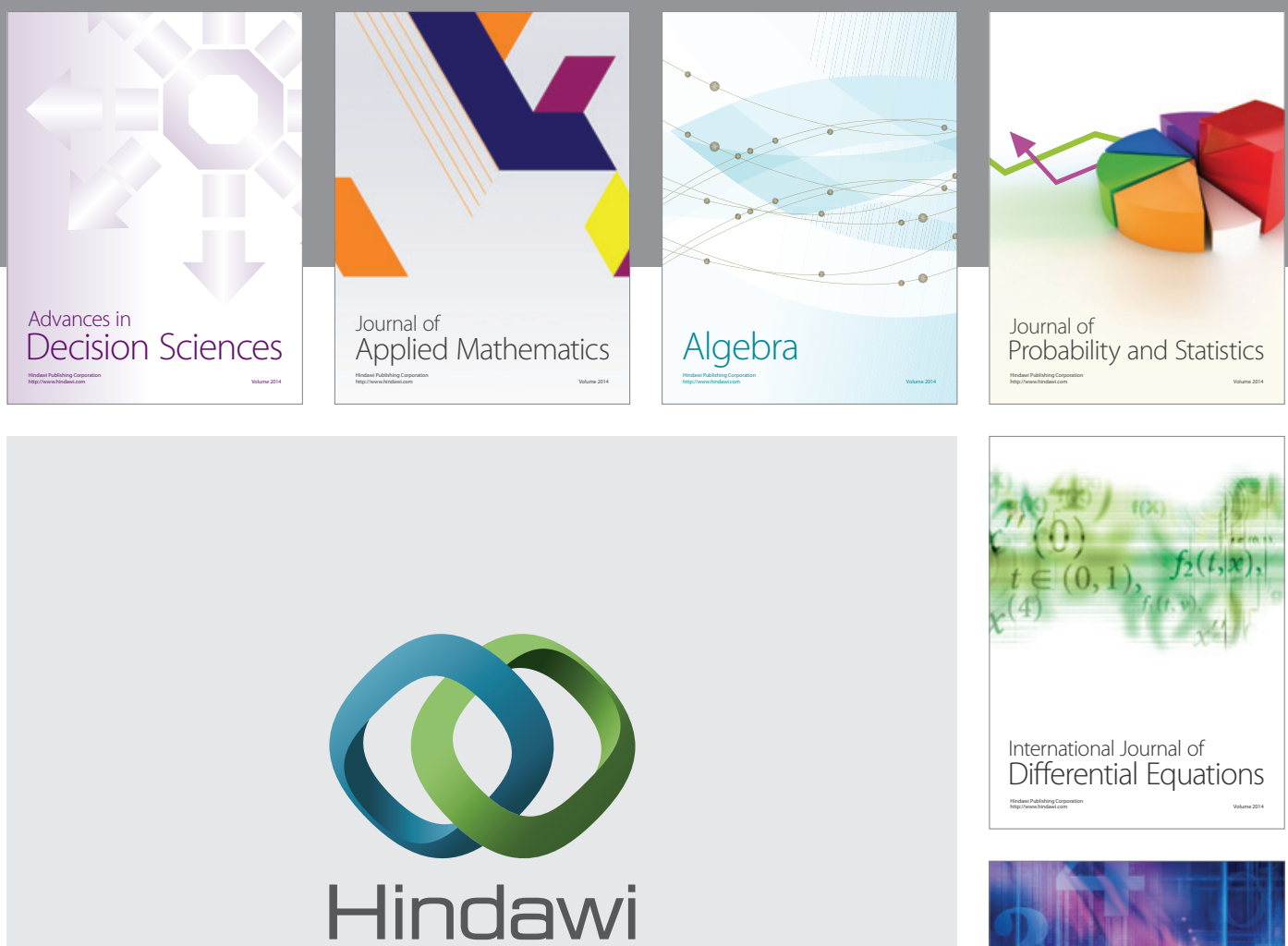

Submit your manuscripts at http://www.hindawi.com
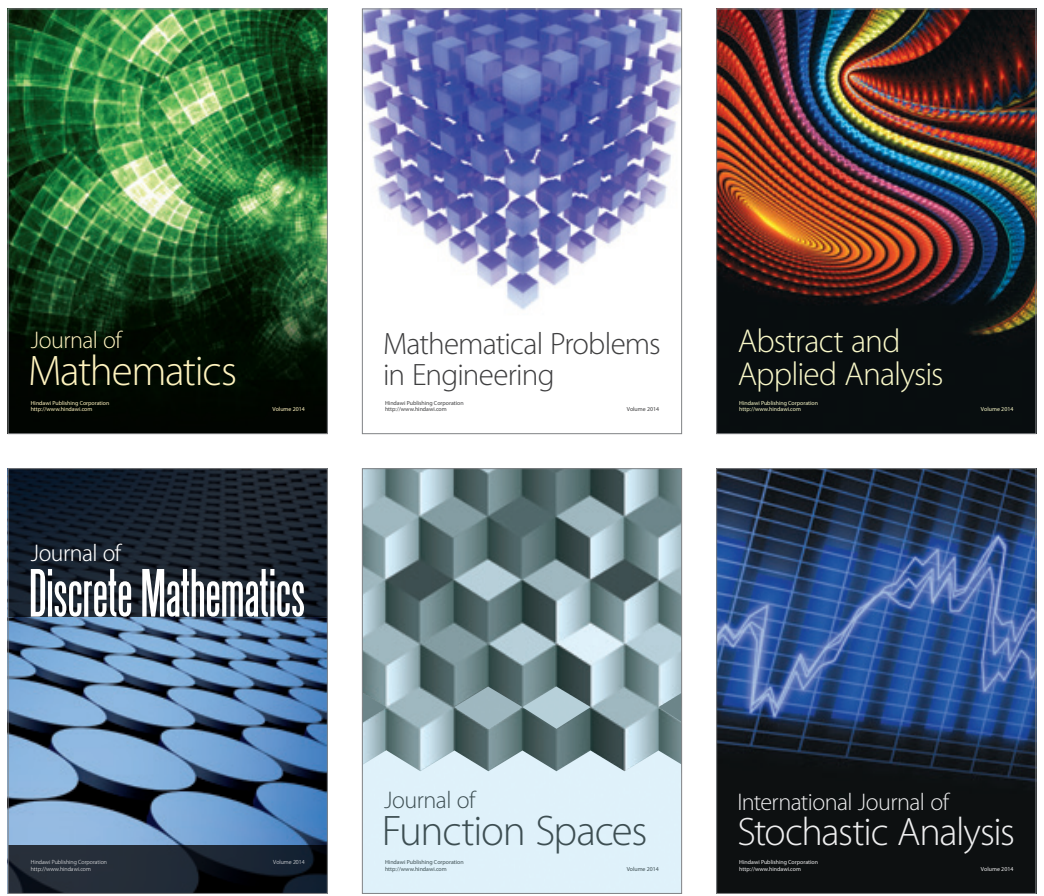

Journal of

Function Spaces

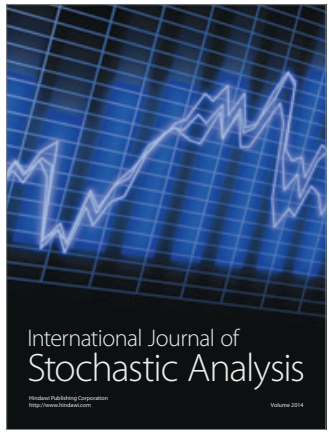

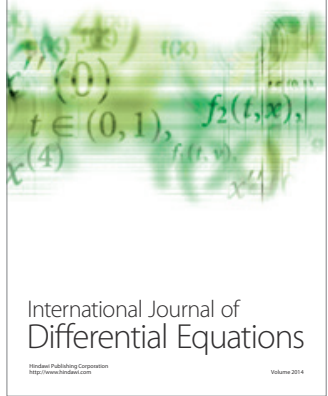
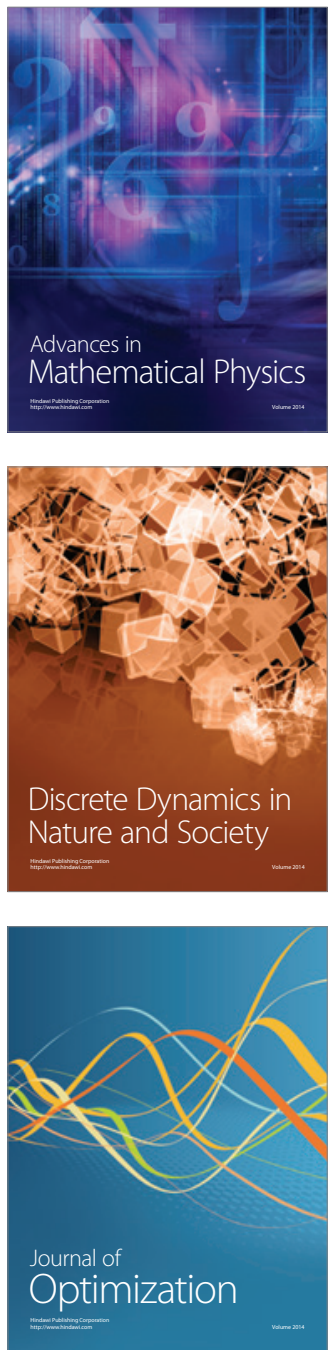